\title{
Feasibility of Fewer Fraction Palliative Radiotherapy in Resource-Limited Setting
}

\author{
Soniya Dulal ${ }^{\mathrm{a}, \mathrm{d}}$, Adeoluwa Akeem Adeniji ${ }^{\mathrm{b}}$, Michael Gary Martin ${ }^{\mathrm{c}}$
}

\section{To the Editor}

Hoskin et al recently reported [1] on the effect of single-fraction versus multi-fraction radiotherapy on ambulatory status among patients with spinal canal compression from metastatic cancer that single-fraction radiotherapy did not meet the criteria for non-inferiority for the primary end point. This was the multicenter non-inferiority randomized clinical trial with 686 patients with life expectancy greater than 8 weeks, and no previous radiotherapy to the same area. The primary end point was ambulatory status at week 8 , based on a four-point scale. The non-inferiority margin for the difference in ambulatory status was $-11 \%$. This study was conducted in two high/ very-high human development index (H/VH-HDI) countries.

As global death rates from communicable diseases fall, deaths from cancer are increasing in low/very-low (L/VL) HDI countries [2]. According to GLOBOCAN 2018, cancer ranks as the first or second most common cause of premature death in over $50 \%$ of countries worldwide.

Radiotherapy is one of the main components of modern cancer treatment and requires substantial capital investment, trained professionals in several disciplines, high precision equipment and a particular external and internal organizational structure. Asia and the Pacific have 30 low middle income countries (LMICs) out of 47 Asia-Pacific countries [3]. More than $50 \%$ of patients requiring radiotherapy in 139 LMICs do not have access to treatment. More alarmingly, the situation is dramatic in low income countries (LICs), where the proportion is higher than $90 \%$ [3].

Radiotherapy has experienced a fast technological advance in the last two decades, which has improved precision in treatment planning and delivery. Use of reduced doses and field of radiotherapy limit the long-term consequences of radiotherapy (i.e. second primary malignancies and cardiovascular mortality) $[4,5]$.

Manuscript submitted February 15, 2020, accepted March 4, 2020

aDepartment of Clinical Oncology, National Academy of Medical Sciences (NAMS), Bir Hospital, Kanti Path, Kathmandu, Nepal

bLagos University Teaching Hospital, Lagos, Nigeria

${ }^{\mathrm{c}}$ West Cancer Center, University of Tennessee, TN, USA

${ }^{\mathrm{d}}$ Corresponding Author: Soniya Dulal, Department of Clinical Oncology, National Academy of Medical Sciences (NAMS), Bir Hospital, Kanti Path, Kathmandu 44600, Nepal. Email: dulalsoniya1@gmail.com

doi: https://doi.org/10.14740/wjon1271
Access to radiotherapy continues to be a common clinical challenge among cancer patients in L/VL-HDI countries. Nigeria has one radiation machine per 19.4 million people versus one machine per 250,000 people in H/VH-HDI countries [6].

Nepal is a developing country with about 29 million persons. Nepal is far below the World Health Organization (WHO) recommendation of one megavoltage machine per million populations which means that Nepal should have at least 29 megavoltage machines [7]. The lack of support in both human and material resources presents significant challenges to delivering safe and timely radiotherapy.

Multi-fraction radiotherapy adds greatly to the overall cost of treatment in addition to the overwhelming workload on the machines with the resultant machines breakdown and can decrease the availability of radiation therapy due to limits in capacity. Twenty-five percent of the Nepalese population and $70 \%$ of the Nigerian population live below the poverty line [8]. Many of these patients present with advanced stage cancer and are from rural areas and often delay or forego treatment because of prolonged wait time and high costs involved in treatment and logistics.

Single-fraction radiotherapy has shown substantial benefits for patient-centric palliative care, including greater quality of life and convenience, economically more feasible, reduced travel time, and total expenditure on travel during radiotherapy is almost half [9].

A prospective study that compared patient-reported outcomes of a broad set of cancer patients with bone metastases demonstrated that single-fraction radiation therapy (SFRT) is equally as effective as multiple-fraction radiation therapy (MFRT) when pain, function and quality of life are considered, according to research presented at the American Society for Radiation Oncology's (ASTRO's) 56th Annual Meeting (September 14 - 17, 2014, San Francisco) [10].

Considering the challenges of making radiotherapy accessible and affordable in developing countries, the International Atomic Energy Agency (IAEA) has also been making the effort to address the problem [11].

Recently, there has been much interest in comparing the treatment outcome from fewer fractions of radiotherapy to the already known multi-fractionated regimens of radiation especially in palliative doses to organs such as spine and radical doses to organs such as prostate, breast and others.

Thus, in the article by Hoskin et al [1], given lack of a difference in overall survival at 12 weeks between the two groups (hazard ratio $(\mathrm{HR})=1.02)$ and the small difference $(-3.5 \%)$ in ambulatory status between the two groups at 8 weeks, single- 
fraction radiotherapy could be practice changing in L/VL-HDI countries by preventing frequent machine breakdown from workload and decreasing costs, thereby ensuring that more people in such countries have access to radiotherapy.

\section{References}

1. Hoskin PJ, Hopkins K, Misra V, Holt T, McMenemin R, Dubois D, McKinna F, et al. Effect of single-fraction vs multifraction radiotherapy on ambulatory status among patients with spinal canal compression from metastatic cancer: the SCORAD randomized clinical trial. JAMA. 2019;322(21):2084-2094.

2. Bray F, Ferlay J, Soerjomataram I, Siegel RL, Torre LA, Jemal A. Global cancer statistics 2018: GLOBOCAN estimates of incidence and mortality worldwide for 36 cancers in 185 countries. CA Cancer J Clin. 2018;68(6):394424.

3. Zubizarreta EH, Fidarova E, Healy B, Rosenblatt E. Need for radiotherapy in low and middle income countries the silent crisis continues. Clin Oncol (R Coll Radiol). 2015;27(2):107-114.

4. Giri U, Patel K, Martin M. Second primary malignancies and cardiovascular mortality in Hodgkin Disease (HD) survivors, a seer database study. International Journal of Radiation Oncology Biology Physics. 2018;102(3):e746.
5. Giri S, Pathak R, Martin MG, Bhatt VR. Incidence of breast cancer among female survivors of Hodgkin lymphoma: a US-population-based trend analysis from 1973 to 2011. Blood. 2015;126(15):1861-1863.

6. Anakwenze CP, Ntekim A, Trock B, Uwadiae IB, Page BR. Barriers to radiotherapy access at the University College Hospital in Ibadan, Nigeria. Clin Transl Radiat Oncol. 2017;5:1-5.

7. Karn A. Current status of availability of radiotherapy machines in Nepal. J Clin Oncol. 2017;35:15_suppl:e18042e18042.

8. Central Intelligence Agency: The World Factbook. https:// www.cia.gov/library/publications/the-world-factbook.

9. Nongkynrih A, Dhull AK, Kaushal V, Atri R, Dhankhar R, Kamboj K. Comparison of Single Versus Multifraction Radiotherapy in Palliation of Painful Bone Metastases. World J Oncol. 2018;9(3):91-95.

10. Conway J, Olivotto I, Miller S, Halperin R, Hoegler $\mathrm{D}$, Beckham W, et al. Patient-reported outcomes on the impact of single versus multiple fraction palliative radiation therapy for uncomplicated bone metastases on pain, function, and degree of symptom distress. International Journal of Radiation Oncology, Biology, Physics. 2014;90(1):S80.

11. Samiei MA. Challenges of making radiotherapy accessible in developing countries. Cancer Control. 2013;2013:83-96. 